\title{
ON THE CONSTANTS ASSOCIATED WITH A REVERSIBLE SUMMABILITY MATRIX ${ }^{1}$
}

M. R. PARAMESWARAN

The matrix $A=\left(a_{n k}\right)$ of real or complex numbers is called reversible if the equations

$$
\sum_{k=0}^{\infty} a_{n k} x_{k}=y_{n} \quad(n=0,1,2, \cdots)
$$

have a unique solution $\left\{x_{k}\right\}$ for each convergent sequence $\left\{y_{n}\right\}$. It is proved in Banach $[1, \mathrm{p} .50]$ that if $A$ is reversible then the equations (1) have their unique solution given by

$$
x_{k}=c_{k} \lim _{n \rightarrow \infty} y_{n}+\sum_{n=0}^{\infty} b_{k n} y_{n} \quad(k=0,1,2, \cdots)
$$

where $c_{k}(k=0,1, \cdots)$ and $b_{k n}(k, n=0,1, \cdots)$ are constants depending only on $A$ and not on the particular sequence $y=\left\{y_{n}\right\}$ chosen. Banach's further statement that $\left\{c_{k}\right\}$ is a bounded sequence was shown to be incorrect by Macphail [3] after Zeller [8] had thrown doubt on the statement. We now discuss some properties of the constants $c_{k}$ and $b_{k n}$ and among other results obtain an extension of the following theorem due to Wilansky [5]:

Theorem A. $A$ reversible conservative matrix $A$ sums a divergent sequence if and only if

$$
\operatorname{lum}_{\mathbf{0} \leq \boldsymbol{k}<\infty}\left\{\left|c_{k}\right|+\sum_{n=0}^{\infty}\left|b_{k n}\right|\right\}=\infty \text {. }
$$

Wilansky's proof, based on Banach's erroneous statement, naturally fails, but Macphail has given a proof without making any assumptions regarding the $c_{k}$ 's.

Theorems 1 and 2 below are extensions of the first part and the second part respectively of Theorem A, while Theorem 5 is an extension of Theorem $\mathrm{A}$ as a whole to any reversible matrix, not necessarily a conservative matrix. Theorems 6 and 7 discuss in the case of a reversible conservative matrix, the effect of the finitude or otherwise of $\|B\| \equiv$ l.u.b. $0 \leq k<\infty) \sum_{n=0}^{\infty}\left|b_{k n}\right|$ on the $c_{k}$ 's.

Received by the editors March 20, 1956 and, in revised form, April 27, 1956.

1 Presented to the 21st Conference of the Indian Mathematical Society, Benares, December, 1955. 
Theorem 1. Any reversible matrix $A$ sums an unbounded sequence if $\|B\|=\infty$.

Proof. It is a classical result that if $\|B\|=\infty$ then there exists a sequence $\left\{y_{n}\right\}$ with limit zero and such that $x_{k}=\sum_{n=0}^{\infty} b_{k n} y_{n} \rightarrow \infty$.

Theorem $2 .^{2}$ If the reversible conservative matrix $A$ sums a divergent sequence, then $\|B\|=\infty$.

Proof. Let us denote the set of equations (1) by the matrix equation $A x=y$ and similarly in other cases. It is known that $A B=I$, the identity [6, Lemma 3]. Now if $\|B\|<\infty$ and $y \in(c)$, where (c) denotes the set of all convergent sequences, then $A(B y)=(A B) y=y$ and hence $x=B y$ is a solution of $A x=y \in(c)$. Since $A$ is reversible, it is the only solution. Since $\|B\|<\infty$ and $y \in(c)$ we see that $x$ is a bounded sequence.

We may now either use the fact that $B$ is actually a conservative matrix, as is proved in [4], and therefore $x=B y$ is a convergent sequence and $A$ does not sum any divergent sequence if $\|B\|<\infty$; or we may use a theorem due to Zeller [9, Satz 7.1] that a conservative matrix which sums only bounded sequences does not sum any divergent sequences.

THEOREM 3. There exist reversible matrices $A$ (necessarily not conservative, by Theorem 2) summing an unbounded sequence and for which $\|B\|<\infty$.

Proof. Take for example $A$ to be the matrix of the transformation

$$
\begin{aligned}
y_{2 m} & =\sum_{p=0}^{m} x_{2 p}, \\
y_{2 m+1} & =x_{2 m+1}+2^{m} \sum_{p=0}^{\infty} x_{2 p} \quad \text { for } m=0,1,2, \cdots .
\end{aligned}
$$

Then

$$
x_{0}=y_{0}, \quad x_{2 m}=y_{2 m}-y_{2 m-2} \quad(m \geq 1)
$$

and

$$
x_{2 m+1}=-2^{m} \lim _{n \rightarrow \infty} y_{n}+y_{2 m+1} \quad(m \geq 0) .
$$

It is seen that $A$ is reversible, $\|B\|=2$ and $c_{2 k}=0, c_{2 k+1}=-2^{k}$, for

2 The referee has pointed out that this result was announced by Wilansky at the February meeting (New York) of the American Mathematical Society. The author thanks the referee for this and other helpful remarks. 
all $k=0,1,2, \cdots$, and that $A$ sums the unbounded sequence $\left\{1,0,0,-2+1,0,-2^{2}+1,0, \cdots,-2^{m}+1,0,-2^{m+1}, 0, \cdots\right\}$.

Theorem 4. If $A$ is reversible and $\|B\|<\infty$, then $A$ sums an unbounded sequence if and only if $\left\{c_{k}\right\}$ is unbounded.

Proof. Let $y=A x \in(c)$. Then

$$
x_{k}=c_{k} \lim _{n \rightarrow \infty} y_{n}+\sum_{n=0}^{\infty} b_{k n} y_{n} .
$$

Also $B y$ is a bounded sequence since $\|B\|<\infty$. Hence $\left\{x_{k}\right\}$ is unbounded if and only if $\left\{c_{k}\right\}$ is unbounded.

The existence of such matrices $A$ is proved by the example given under the previous theorem.

Combining Theorems 1 and 4 we get the

THEOREM 5. The reversible matrix $A$ sums an unbounded sequence if and only if

$$
\begin{gathered}
\text { either }\|B\|=\infty \\
\text { or }\left\{c_{k}\right\} \text { is unbounded. }
\end{gathered}
$$

THEOREM 6. If $A$ is a reversible conservative matrix and $\|B\|<\infty$ then

(a) $c_{k}=0$ for all $k$, and (b) $A$ is of type $M$.

Proof (a). As in the proof of Theorem 2, $x=B y$ is the only solution of $A x=y$, for all $y \in(c)$. This implies by (2) that $c_{k} \equiv 0$.

(b) This is a consequence of Theorem 2 and the fact that a reversible conservative matrix not of type $M$ will sum a divergent sequence, [2, Theorem 3].

TheOREM 7. There exist reversible conservative matrices $A$ with $\|B\|$ $=\infty$ and satisfying also any one of the following conditions:

(a) $c_{k}=0$ for all $k$.

(b) $A$ is of type $M$.

(c) $c_{k} \rightarrow \infty$ when $k \rightarrow \infty$.

(d) $\left\{c_{k}\right\}$ is bounded and $c_{k} \neq 0$ for any $k$.

Proof. We have the following examples.

(a), (b): The matrix of $(C, 1)$ summability is a well-known example.

(c): We take $A$ to be the matrix of the transformation given by

and

$$
y_{2 m}=\sum_{p=0}^{m} \frac{x_{2 p}}{8^{p}}+\frac{1}{2^{m}} \sum_{p=0}^{\infty} \frac{x_{2 p}}{8^{p}}
$$




$$
y_{2 m+1}=\sum_{p=0}^{\infty} \frac{x_{2 p}}{8^{p}}-\frac{x_{2 m+1}}{2^{2 m+1}} \quad \text { for } m=0,1,2, \cdots .
$$

Then it is easily verified that $A$ is reversible, $\|B\|=\infty, c_{0}=-1$ and $c_{k}=2^{k}$ for $k=1,2,3, \cdots$.

(d) If we take $A$ as the matrix of the transformation

$$
y_{2 m}=\sum_{p=0}^{m} \frac{x_{2 p}}{2^{p}}+\frac{1}{2^{m}} \sum_{p=0}^{\infty} \frac{x_{2 p}}{2^{p}}
$$

and

$$
y_{2 m+1}=\sum_{p=0}^{\infty} \frac{x_{2 p}}{2^{p}}-x_{2 m+1}, \quad \text { for } m=0,1,2, \cdots
$$

then $A$ is reversible, $\|B\|=\infty, c_{0}=-1$ and $c_{k}=1$ for $k \geq 1$.

REMARK. The conservative matrix $A$ is said to be co-regular if $\lim _{n \rightarrow \infty} \sum_{k=0}^{\infty} a_{n k}-\sum_{k=0}^{\infty} \lim _{n \rightarrow \infty} a_{n k} \neq 0$, and co-null otherwise. Wilansky and Zeller [7] have proved that a coregular reversible matrix $A$ has $c_{k}=0$ for all $k$. The examples under (c) and (d) above show that for co-null matrices none of the $c_{k}$ 's need vanish and that it is even possible that $\lim _{k \rightarrow \infty} c_{k}=\infty$.

\section{REFERENCES}

1. S. Banach, Théorie des opérations linéaires, Warsaw, 1932.

2. J. D. Hill, Some properties of summability. II, Bull. Amer. Math. Soc. vol. 50 (1944) pp. 227-230.

3. M. S. Macphail, A remark on reversible matrices, Proc. Amer. Math. Soc. vol. 5 (1954) pp. 120-121.

4. M. R. Parameswaran, On the reciprocal of a $K$-matrix, J. Indian Math. Soc. vol. 20 no. 4 (1956).

5. A. Wilansky, A necessary and sufficient condition that a summability method be stronger than convergence, Bull. Amer. Math. Soc. vol. 55 (1949) pp. 914-916.

6. - Convergence fields of row-finite and row-infinite reversible matrices, Proc. Amer. Math. Soc. vol. 3 (1952) pp. 389-391.

7. A. Wilansky and K. Zeller, Inverses of matrices and matrix transformations, Proc. Amer. Math. Soc. vol. 6 (1955) pp. 414-420.

8. K. Zeller, FK-Räume and Matrixverfahren, Math. Zeit. vol. 58 (1953) pp. 46-48.

9. - Allgemeine Eigenschaften von Limitierungsverfahren, Math. Zeit. vol. 53 (1951) pp. 463-487.

Ramanujan Institute of Mathematics, Madras 